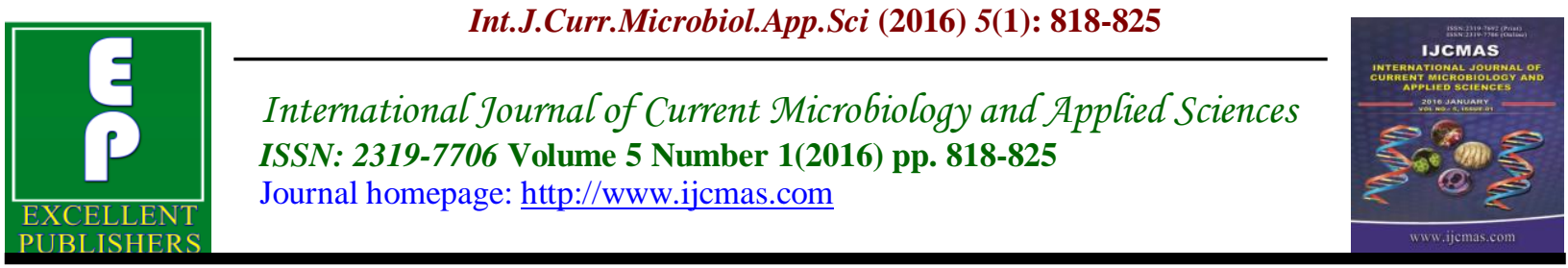

Original Research Article

http://dx.doi.org/10.20546/ijcmas.2016.501.084

\title{
Assessment of Frequency of Long Bone Osteomyelitis in Traumatic Patients Undergoing Orthopedic Surgery in Imam Reza (AS) Hospital-Tabriz
}

\author{
Mohammad Irajian ${ }^{1}$ and Arman Beheshtirooy ${ }^{2} *$ \\ ${ }^{1}$ Department of Orthopedic, Faculty of Medicine, Tabriz University of Medical Sciences, \\ Tabriz, Iran \\ ${ }^{2}$ Medical Student, Faculty of Medicine, Islamic Azad university Tabriz branch, Tabriz, Iran \\ *Corresponding author
}

\section{Keywords \\ Osteomyelitis, Open Fractures, Closed Fractures, Antibiotics, Trauma.}

\section{Article Info}

Accepted:

20 December 2015 Available Online: 10, January 2016
A B S T R A C T

Despite significant progress in the field of infectious diseases, osteomyelitis remains a problem for the orthopedic community. Rate of infection in open fractures in long bones is $64-4 \%$ mentioned. Chronic infections have serious side effects, including disability and even amputation, and still is not a desirable treatment. This study aimed to assess the incidence of osteomyelitis in Imam Reza hospital of Tabriz and its influencing factors and methods to reduce and prevent osteomyelitis is done in traumatic patients. All cases of traumatic patients with long-bone fractures who is admitted to Imam Reza hospital from March 2010 until the end of December 2013 extracted. Recorded variables are age, sex, multiple trauma or trauma of single organs, from admission until orthopedic surgery time, fracture type (open or closed), received prophylactic antibiotics before and after surgery, systemic diseases were analyzed. 151 of the 184 patients were male $(82.1 \%)$ and 33 were female $(17.9 \%)$.Fractures in 73 patients with a frequency of $39 \%$ was open and in 111 patients was close (60\%).111 of the patients had associated diseases that were respectively 47 patients ICU hospitalization (25\%),43 patients surgery $(23 \%)$ and 9 patients diabetes (4\%).Only 24 patients with frequency of $13 \%$ were suffering from osteomyelitis. The relationship between delay in surgery was significantly associated with osteomyelitis ( $p$-value $<0.01)$.The study not found any significant relationship between age and osteomyelitis $(\mathrm{p}$-value $=0.79)$. The relationship between sex and osteomyelitis, were significant which represents an increase of osteomyelitis in male sex (p-value $<0.01)$. The relationship between type of fracture and osteomyelitis were significant, which increases in open fractures ( $\mathrm{p}$-value <0.01). The relationship between systemic diseases associated with osteomyelitis was significant ( $\mathrm{p}$-value $<0.01$ ). There is no relationship between receiving antibiotics before surgery and osteomyelitis $(\mathrm{p}$-value $=0.67)$. The relationship between receive antibiotics after surgery and osteomyelitis was significant ( $\mathrm{p}$-value $<0.01$ ). According to the study it can be concluded that systemic disease, older age, open fracture, In Males and a delay in orthopedic surgery in traumatic patients increases osteomyelitis and take antibiotics after surgery reduce the risk of osteomyelitis. 


\section{Introduction}

Hippocrates first described this disease and recommended splinting, applying a clean bandage for open fractures, and identifying risk factors of osteomyelitis (1). Although infection may occur after any surgery, the incidence of infection is greater in patients with high-energy fractures and in those with impaired immune systems. Osteomyelitis is one of the major challenges in orthopedic surgery. Despite advances in the field of infectious diseases in the past decades, osteomyelitis has remained as a problem for the orthopedic community. Bones are a unique shelter for microorganisms to produce the biofilm allowing them to flexibly be connected to biological and implanted surfaces which have remained insensitive to the host defense system (1). Research findings indicate that osteomyelitis is generally associated with dead and infected implant bone formation under the used device. Sequestrum is an infected necrotic bone whose blood flow communication with adjacent tissues is destroyed and is the symptom of chronic osteomyelitis and infection will continue in its presence. Due to the fact that some bacteria can form a biofilm on implant surface and hide themselves from phagocytosis or antibiotic, the presence of infection in implant makes treatment more difficult to be achieved (2 and 3).

Treatment of chronic infections is both expensive and challenging. Moreover, the cure rate is still unfavorable. In an ideal and favorable condition, therapeutic methods must provide a technique for complete resolution of infection, optimal performance, and proper mobility of the infected limbs (4).

Treatment also aims at controlling infection, converting an infectious nonunion into non- infection nonunion, and finally resolving the nonunion problem. The presence of cureresistant bacteria or loose implant is indicative of a need to remove the implant (5).

Now, despite advances in both surgical treatment and pharmacotherapy, recurrence rate of bone infection is between $20 \%$ and $30 \%$ (4). Infections that occur after fracture surgery are exogenous, i.e. the origin of contamination comes from outside the body and the source of these infections can be initial trauma and fracture wound (open fracture) or intraoperative or postoperative infection. (6)

Reasons for choosing this topic include the fact that no study has been conducted in this hospital center to determine the frequency of osteomyelitis, its influencing factors, and techniques to be used in order to reduce and to prevent osteomyelitis in trauma patients and the statistical comparison with other findings should also be performed. The aim of this study was to evaluate the frequency of osteomyelitis of long bones in trauma patients of Imam Reza Hospital.

\section{Methods and Materials}

In a descriptive-cross sectional study conducted in Tabriz on patients with fractures, the frequency of osteomyelitis of long bones in trauma patients of Imam Reza Hospital was studied.

The sample consisted of trauma patients hospitalized in Imam Reza hospital of Tabriz. They had fractures of long bones during the period of 2010- 2013 and underwent fixation surgery in different ways. Then, during the follow-up period, they suffered from osteomyelitis of long bones and, finally, 184 patients participate in the study. 


\section{Inclusion Criteria}

All patients with trauma who had orthopedic surgery in Imam Reza Hospital, Tabriz. Those who suffered long bone fractures after trauma and orthopedic surgery, during 20102012, participated in this study.

\section{Exclusion Criteria}

1.Patients who passed away during hospitalization.

2.Patients who underwent limb amputation during hospitalization due to the severity of trauma.

3.Patients with non-invasive and closereduction measures.

4.Patients who were sent to Shohada Hospital during orthopedic consultation to receive orthopedic measures.

5.Patients whose files were not complete.

6. Patients who had left the hospital with personal consent.

\section{Ethical Considerations}

Demographic information collected from the participants was anonymously coded and used in this study. No personal information of these patients has entered in this study and their statistical analysis is collectively expressed.

No one (except those involved with the project) had accessed to information collected in this study and there was no abuse of such information.

Collected information was recorded as facts without any bias or selection of special patients to reach the desired outcome. In order to avoid biases, assumptions were written in bilateral and open form. To achieve and record the results, there was no bias in the selection of statistical methods and their analysis.

Due to lack of specific therapeutic intervention and retrospective study of the patients, there was no need to have consent from filled out by patients.

\section{Statistical Analysis}

The collected data were analyzed by SPSS17 statistical software. The collected data were expressed as percentage and mean \pm SD. Continuous (quantitative) variables were compared by Independent samples and Paired t test. Categorical (qualitative) variables were compared by contingency tables and Chi-square test or Fisher's exact test. P-value $\leq 0.05$ was considered statistically significant.

\section{Results and Discussion}

One hundred and eighty-four trauma patients hospitalized in Imam Reza hospital of Tabriz were studied. They had fractures of long bones and orthopedic surgery during the period of 2010- 2013.

In this study, of 184 patients, there were 75 persons (40\%) below 20 years old, 54 patients (29\%) between 39-21 years, 39 patients $(21 \%)$ between 54-40 years, 12 patients (6\%) between 74-55 year and 4 patients (2\%) above 74 years of age. Considering gender, 151 patients $(1.82 \%)$ and $33(9.17 \%)$ were male and female, respectively.

Regarding open fractures in trauma patients with osteomyelitis of long bones, 73 persons (39\%) and 111 patients (61\%) suffered from open fractures and closed fractures, respectively. 
One hundred and eleven patients had a history of co-morbid diseases including hospitalization in ICU (47 persons $=25 \%$ ), recent surgery (43 patients $=23 \%$ ), diabetes (9 patients $=4 \%), \quad$ receiving immunosuppressive drugs for more than a week (7 patients $=3 \%)$, hypothyroidism $(1$ person $=.5 \%)$ and 4 patients $(2 \%)$ were affected by other diseases.

One-hundred eighty-four patients (89\%) with long bone fractures had a history of taking antibiotics before surgery. All patients who did not receive prophylactic antibiotics before surgery had closed fractures.

$176(95 \%)$ patients with long bone fractures had taken antibiotics after surgery. Of 184 patients, 3 persons in less than 24 hours after trauma, 8 persons on the second day after trauma, 11 persons on the third day, 48 persons from 4-7 days after trauma, and 114 persons over more than a week after trauma had orthopedic surgery.

Only 24 persons (13\%) were suffering from osteomyelitis. There was a significant relationship between increased time delay in surgery and osteomyelitis $(\mathrm{P}<.01)$.

There was no significant relationship between age and osteomyelitis $(\mathrm{P}=.79)$. There was a significant correlation between gender and osteomyelitis representing an increase of osteomyelitis in males $(\mathrm{P}<.01)$.

There was a significant correlation between the type of fracture and osteomyelitis indicating an increase in the incidence of osteomyelitis for open fractures $(\mathrm{P}<.01)$.

There was a significant correlation between systemic diseases and osteomyelitis, so that these patients had greater rate of hospitalization in ICU and diabetes $(\mathrm{P}<.01)$.
No significant relationship was observed between taking antibiotics before surgery and osteomyelitis $(\mathrm{P}=.67)$. There was a significant correlation between osteomyelitis and receiving antibiotics after surgery $(\mathrm{P}$ $<.01)$.

Osteomyelitis is one of the major challenges in orthopedic surgery. Osteomyelitis involves the cooperation of several teams and each patient's preparation for surgery and medical treatment. Although infection may occur after any surgery, the incidence of infection is greater in patients with highenergy fractures and in those with impaired immune systems.

Underlying factors leading to infection after fracture surgery include openness of fractures, soft tissue injuries, smoking, diabetes, age and use of immunosuppressive drugs.

In the present study, the frequency of osteomyelitis in surgical patients was $13 \%$ and in comparison with globally reported relative frequency of $4-64 \%$, the frequency observed indicates the desirable status of osteomyelitis in this center.

In this study, a significant correlation was observed between osteomyelitis and surgical delays for fractures of long bones $(\mathrm{P}<.01)$ and increasing the delay duration in surgery led to osteomyelitis. However, Mohammadi et al. did not find any correlation between the time of surgery and the incidence of osteomyelitis in their study. The findings of this study can be due to several factors including adjacent tissue infections and its potential for further expansion in the bone over time, the possibility of nosocomial infection during hospitalization from hospital environment including the emergency department and ICU. 
In spite of correlation observed in this study, the correlation was non-significant according to Cramer's- $\mathrm{V}<0.01$ indicating the potential role of other factors in increasing the incidence of osteomyelitis. In this study, there was no significant relationship between age and osteomyelitis $(\mathrm{P}=.79)$.

In the present study, there was a significant correlation between gender and osteomyelitis representing an increase of osteomyelitis in males $(\mathrm{P}<.01)$. This is consistent with previous study(7-10) in which, of 60 patients being infected after surgery, 49 patients $(81.7 \%)$ and 11 patients $(18.3 \%)$ were male and female, respectively. This consistency indicates the differences of hormonal and genetic impacts on host immune system and susceptibility to osteomyelitis.

Table.1 Correlation between Time and Osteomyelitis

\begin{tabular}{lcccccc}
\hline & \multicolumn{5}{c}{ Time(hour) } & \multirow{2}{*}{ Total } \\
\hline With Osteomyelitis & $12-24$ & $24-48$ & $48-72$ & $72-168$ & $>168$ & \\
$\begin{array}{l}\text { Without } \\
\text { Osteomyelitis }\end{array}$ & 1 & 3 & 3 & 5 & 9 & 24 \\
\hline Total & 3 & 8 & 11 & 48 & 114 & 184 \\
\hline
\end{tabular}

Table.2 Correlation between Age and Osteomyelitis

\begin{tabular}{lcccccc}
\hline & \multicolumn{5}{c}{ Age (year) } & \multirow{2}{*}{ Total } \\
\hline With Osteomyelitis & 15 & 2 & 6 & 1 & 0 & 24 \\
Without & 60 & 52 & 33 & 11 & 4 & 160 \\
Osteomyelitis & 75 & 54 & 39 & 12 & 4 & 184 \\
\hline Total & & & & & \\
\hline
\end{tabular}

Table.3 Correlation between Underlying Diseases and Osteomyelitis

\begin{tabular}{|c|c|c|c|c|c|c|c|c|}
\hline & \multicolumn{7}{|c|}{ Underlying diseases } & \multirow[b]{2}{*}{ Total } \\
\hline & $\begin{array}{c}\text { No } \\
\text { Diseases }\end{array}$ & $\begin{array}{l}\text { Diabetes } \\
\text { Mellitus }\end{array}$ & Immunodeficiency & $\begin{array}{l}\text { Thyroid } \\
\text { Diseases }\end{array}$ & $\begin{array}{c}\text { Other } \\
\text { surgery }\end{array}$ & $\begin{array}{l}\text { Admitted } \\
\text { in ICU }\end{array}$ & Others & \\
\hline $\begin{array}{l}\text { With } \\
\text { Osteomyelitis }\end{array}$ & 5 & 2 & 1 & 0 & 5 & 10 & 1 & 24 \\
\hline $\begin{array}{l}\text { Without } \\
\text { Osteomyelitis }\end{array}$ & 68 & 7 & 6 & 1 & 38 & 37 & 3 & 160 \\
\hline Total & 73 & 9 & 7 & 1 & 43 & 47 & 4 & 184 \\
\hline
\end{tabular}


In this study, there was a significant relationship between the type of fracture and osteomyelitis indicating an increase in the incidence of osteomyelitis for open fractures $(\mathrm{P}<.01)$. This finding is in line with the findings of previous study(7-10). In their study, 39 out of 60 studied cases had had follow-up fixation surgery in open fractures. In other words, there was $17.41 \%$ osteomyelitis in open fractures which has been raised by increasing the amount of contamination in fractures, the severity of tissue damage, loss of skin barrier, and inadequate tissue perfusion. These factors may increase the risk of infection.

Considering the relationship between systemic diseases and osteomyelitis in this study, there was a significant relationship between systemic diseases and osteomyelitis $(\mathrm{P}<.01)$, so that the incidence of ICU hospitalization and diabetes $(8.3 \%)$ was more in these people. This finding is consistent with the study conducted by previous study(7-10). In their study, diabetes was observed in 6 cases $(8.6 \%)$.

No significant relationship was observed between taking antibiotics before surgery and osteomyelitis $(\mathrm{P}=.67)$. It may be due to lack of adequate number of patients who did not take antibiotics before surgery and the studied patients just included patients who had taken antibiotics. Hence, no appropriate conclusion can be draw in this regard. However, in this study, taking antibiotics after surgery was significantly associated with osteomyelitis $(\mathrm{P}<.01)$.

In a research study, Olsen et al. examined risk factors of infection after orthopedic spine surgeries. The overall rate of infection after spine surgery within five years of the study was $2 \%$. Diabetes was independently correlated with infection after spine surgery. High preoperative or postoperative serum glucose levels are independently correlated with an increased infection risk. Injecting prophylactic antibiotics within one hour before surgery and increasing the antibiotics dose to adjust the dose in obesity were also important strategies for reducing the infection risk after spine surgery. (11) In comparison with the present study, as the findings suggest, $4 \%$ of patients with osteomyelitis were affected by diabetes and this is consistent with Olsen's study. In present study, no sufficient finding in favor of using prophylactic antibiotics to prevent osteomyelitis was found so it cannot be compared with other studies.

Charalambous examined the impact of delay in surgery on open tibia fractures and osteomyelitis. The amount of infection and secondary fusion surgery were compared in two groups using statistical methods. No statistically significant difference was observed between early and delayed treatment groups. The study states that they failed to show a significant difference for the amount of infection or the need for secondary surgery between early- and delayed-surgical treatments of open tibia fractures. (12) Although, in the present study, there was a significant relationship between delay in surgery of fractures in long bones and osteomyelitis and the more delay in surgery was consistent with more osteomyelitis ( $\mathrm{P}<.01)$, the impact and correlation coefficient for this variable was low according to Cramer's- $\mathrm{V}<0.01$ and no great impact is considered. However, given the p-value, findings are significant and the above factors and variable along with other factors such as diabetes and other nonidentified factors may be more effective.

In one study conducted by Pollak et al. on the relationship between debridement time and osteomyelitis in trauma patients undergoing orthopedic surgery, no 
conclusion was drawn and they stated that there was no relationship between delay in surgical debridement and being affected by osteomyelitis (13). However, the results of the present study were not in line with theirs and a significant correlation was found between surgery time and being affected by osteomyelitis.

Regarding the results of the present study indicating the effect of surgical delay on osteomyelitis and opposite findings of other studies, one of the factors leading to such controversy can be attributed to the probability of osteomyelitis and contamination in bones during hospitalization inside the section and the hospital source of infection which needs to investigate the strains isolated from osteomyelitis.

In conclusion, according to results indicating the reduction of osteomyelitis through reducing the time from trauma to surgery, in order to minimize the risk of osteomyelitis, the treatment system of trauma patients with fractures can be provided in a coherent form through minimizing trauma -surgery period.

Since the frequency of osteomyelitis in surgical patients was $13 \%$ and in comparison with globally reported relative frequency of 4-64\%, the frequency observed indicates the desirable status of osteomyelitis in Imam Reza Hospital, Tabriz, some measures in terms of improving operating room sterilization, proper hygiene measures by the emergency system before and after reception in emergency department and while sending the patient into the hospital reception center can be taken in order to further reduce osteomyelitis and to reduce the side effects.

The suggestions includes, with regard to a large number of cases with incomplete files ignored in this study, it is suggested that registration of medical records including background as operation information are provides as much as possible in a complete and typed format.

Further exploration on the incidence of osteomyelitis in a variety of fixation methods is suggested for future studies.

Considering that no study has been conducted on the potential sources of infection and nosocomial infection in osteomyelitis, it is suggested that more studies be carried out on existing strains in emergency department, orthopedic ward, and ICU as well as strains isolated from the infection to compare and determine the possible contribution of each.

Due to the lack of alignment between the current research and the research presented on the impact of surgical delay on osteomyelitis, other research can be carried out in this regard. Investigating strains isolated from patients with osteomyelitis, one of the possible causes of the surgical delay impact on osteomyelitis in the present study can be expressed.

\section{References}

1.Lew DP, Waldvogel FA. 2004. Osteomyelitis. Lancet, 364:369-379.

2.Costerton J W, Stewart PS, Greenberg EP. 1999. Bacterial biofilms: a common cause of persistent infections. Science, 284 (5418), 1318-22.

3.Stewart PS, Costerton JW. 2001. Antibiotic resistance of bacteria in biofilms. Lancet, 358 (9276), L1358.

4.Conterno LO, da Silva Filho CR. 2009. Antibiotics for treating chronic osteomyelitis in adults. Cochrane Database Syst Rev, 3,1-30.

5.Trampuz A, Zimmerli W. 2006. Diagnosis and treatment of infections 
associated with fracture fixation devices. Inter J Care Injured, 37, S59-66.

6.Chapman MW. 2001. Chapman's Orthopaedic Surg. 3th edition. Lippincott Williams \& wilkins,: $3505-14$

7.Schenker ML, Yannascoli S, Baldwin KD, Ahn J, Mehta S. 2012. Does timing to operative debridement affect infectious complications in open long bone fractures? A systematic review. J Bone Joint Surg Am, 94,1057-1064.

8.Penn-Barwell JG, Bennett PM, Fries CA, Kendrew JM, Midwinter MJ, Rickard RF. 2013. Severe open tibial fractures in combat trauma: management and preliminary outcomes. Bone Joint J, 95,101-105.

9.Calhoun JH, Manring MM, Shirtliff M. 2009. Osteomyelitis of the long bones. Semin Plast Surg, 23(2),5972.

10.Waldvogel FA, Medoff G, Swartz MN. 1970. Osteomyelitis: A review of clinical features, therapeutic considerations, and unusual aspect. $N$ Engl J Med, 282,198-206.

11.Olsen, M. A., Nepple, J. J., Riew, K. D., Lenke, L. G., Bridwell, K. H., Mayfield, J., \& Fraser, V. J. 2008. Risk factors for surgical site infection following orthopaedic spinal operations. The Journal of Bone \& Joint Surgery, 90(1), 62-69.

12.Charalambous, C. P., Siddique, I., Zenios, M., Roberts, S., Samarji, R., Paul, A., \& Hirst, P. 2005. Early versus delayed surgical treatment of open tibial fractures: effect on the rates of infection and need of secondary surgical procedures to promote bone union. Injury, 36(5), 656-661.

13.Pollak, A. N., Jones, A. L., Castillo, R. C., Bosse, M. J., MacKenzie, E. J., \& LEAP Study Group. 2010. The relationship between time to surgical debridement and incidence of infection after open high-energy lower extremity trauma. The Journal of Bone \& Joint Surgery, 92(1), 715.

\section{How to cite this article:}

Mohammad Irajian and Arman Beheshtirooy. 2016. Assessment of Frequency of Long Bone Osteomyelitis in Traumatic Patients Undergoing Orthopedic Surgery in Imam Reza (AS) Hospital-Tabriz. Int.J.Curr.Microbiol.App.Sci. 5(1): 818-825. http://dx.doi.org/10.20546/ijcmas.2016.501.084 